\title{
Preparation of Gold Nanoparticles Loaded Chitin Nanofiber Composite
}

\author{
Zameer Shervani $^{1 *}$, Yukawa Taisuke ${ }^{1}$, Shinsuke Ifuku ${ }^{1}$, Hiroyuki Saimoto ${ }^{1}$, Minaru Morimoto ${ }^{2}$ \\ ${ }^{1}$ Department of Chemistry and Biotechnology, Graduate School of Engineering, Tottori University, Tottori, Japan \\ ${ }^{2}$ Research Center for Bioscience and Technology, Tottori University, Tottori, Japan \\ Email: 'zameer@chem.tottori-u.ac.jp, shervani.nanotec@gmail.com
}

Received August 23, 2012; revised September 30, 2012; accepted October 9, 2012

\begin{abstract}
Chitin nanofibers (CNFs) processed from second most abundant biomolecule chitin were loaded with gold metallic nanoparticles (Au NPs) to obtained hybrid organic-inorganic composite which was molded in different forms dispersion, flakes, powder, and transparent thin film. First pre-organized Au NPs were prepared in soluble triblock copolymer poly (methyl vinyl ether) (PMVE) by reduction of gold precursor salt $\left(\mathrm{HAuCl}_{4} \cdot 3 \mathrm{H}_{2} \mathrm{O}\right)$ by $\mathrm{NaBH} 4$ then NPs were mixed with diluted CNFs suspension to obtain CNFs-Au NPs composite. CNFs of width 25 - $40 \mathrm{~nm}$ were prepared by combination of chemical and mechanical processing in wet acidic condition from $1 \mathrm{wt} \%$ crab shell chitin slurry. When polymer stabilized Au NPs blended with CNF suspension, all Au NPs and 56\% polymer were found mass transferred from water phase to entangle with more polar moieties of chitin. Composite's suspension and compressed dried film were characterized by recording digital images, UV-vis, TEM, SEM, and XRD spectroscopies. When $70 \mathrm{~m}$ thin non transparent composite film was impregnated with tricyclodecane dimethanol dimethacrylate (TCDDMA) resin and subsequent polymerized with photoinitiator 2-hydroxy-2-methylpropiophenone, the film became transparent due to filling of nanosized CNFs in the cages of the resin.
\end{abstract}

Keywords: Chitin Nanofibers; CNFs-Gold Nanocomposites; Gold Nanopartilces; Soluble Polymer

\section{Introduction}

Chitin nanofibers (CNFs) are extracted from crab and prawn shells [1,2]. CNFs are composed of chitin compound. Chitin in powder form is obtained from fish industry which is otherwise thrown as industrial waste. Chitin is second most plentiful biomaterial [3] next to cellulose exists on earth with yearly production of 1011 tons. Chitin raw dried powder is manufactured from exoskeleton of sea food shellfish, crabs, shrimps, and insects. Chitin content in fish industrial waste is $8 \%-33 \%$, which is thrown if not used. Since CNFs are biodegradable having typical width 10 - $20 \mathrm{~nm}$ and large surface-to-mass ratio thus they are being prepared, studied, and applied more recently world wide along with rapidly growing field nanotechnology dealing with the better properties of materials when their sizes are reduced in the range $1-100$ $\mathrm{nm}$. Fibrilated chitin in the form of highly viscous gel suspension in water has found scope in pharmaceuticals [4], chiral separation [5], fillers in silsesquioxane cages [6]. When blended with inorganic metals to prepare advanced hybrid organic-inorganic [7] composites they can have applications in electronics, electrical, optical de-

${ }^{*}$ Corresponding author. vices and much needed solar energy production. Thus our group is actively engaged in developing chitin research to make a number of products from atomized or fibrillated chitin in the form of CNFs and its derivatives [811]. Chitin obtained from its natural resources is highly crystalline and most of it is chitin conformation though the contents of $\alpha$-, $\beta$-, and $\gamma$-chitin depends on the source. We have published a number of review articles $[9,12,13]$ covering back ground of CNFs in detail: method of preparation, sources, composition, physical and chemical properties, characterization, composites and derivatives preparations, and surface modification. For atomization or fibrillation of $1 \mathrm{wt} \%$ wet chitin to CNFs three types of methods are being used and compared. A very recently developed [8] Star Burst atomization system which employed high pressure water jet system where slurry of chitin in high acetic acid medium is introduced in chamber of Star Burst system machine where it is fibrillated into NFs of width 18.0 - $19.0 \mathrm{~nm}$. Atomizataion occurred in this newly developed machine chamber by a very high speed rotating ceramic ball that throws out fine fibrillated NFs at extremely high pressure of $245 \mathrm{MPa}$ through an outlet nozzle. The two other common apparatus used for fibrillation are blender and grinder. The advantages of 
Star Burst system over blender or grinder for fibrillation have been described in article [8] published recently. In review article [9] molecular structure of chitin, hierarchical organization on the surface of crab shell exoskeleton and isolation from crab and prawn shell has been described. Method of isolation of CNFs from crab or prawn shell using a number of chemical treatments followed by grinder treatment has been explained. After successfully establishing high technologies to isolate CNFs, we started developing CNFs research to produce CNFs-inorganic nanometal composites to have scope in electrical, electronics, and optical devices and pharmaceutical applications. CNFs-nanometallic composites have double dose effect when NFs are conjugated with antibacterial metallic nanoparticles. Following text introduces the importance of organic-inorganic hybrid functional materials to justify our research of CNFs-Au NPs composite preparation, characterization, and applications. A number of organic-inorganic products have been developed as described followings but non have addressed the issues we are interested in do develop low cost, easy preparation at ambient conditions and short processing time. In a review article [14] authors have shown attaching different functional groups for delivery of drugs, DNA, protein, drug targeting moieties lipid and carbohydrate to low toxicity Au NPs core. Authors have cited advances in Au NPs monolayer surface with controlled particles size and tunable surface properties for efficient delivery of drugs and biomolecules that have been achieved. Nguyen et al. [15] have conducted a detailed survey on the accomplished work till recently for the ability of Au NPs for immobilization of biomolecules while retaining their bioactivities is a major advantage to apply them as biosensors. Research of biomolecules conjugated Au NPs biosensors has reached to the stage of practical application. Modification of NPs of controlled size by conjugating them with polymers, cell penetrating peptides, contrast agents, and specific targeting ligands has been achieved. Funtionalization of Au NPs for sensitive and selective biosensors for detection of targeted of metal ions, organic molecules, proteins, RNA, DNA, other cells having optical, electrical, and electrochemical signals have also been demonstrated. Development and testing of Au nanoprobes for nucleic acid, proteins, and cell imaging have also been described in the above review article. Au NPs have gained prominence in drug targeting when Etame et al. [16] showed experimentally the enhanced permeation of Au NPs involving systemic cancers. This has allowed researchers to expand the application of NPs for passive targeting of malignant tumors. The permeation of particles $4-24 \mathrm{~nm}$ size and PEG chain lengths of molecular weight 1000 - 10000 were investigated. Laser ablation and electrospinning techniques have been applied [17] to prepare poly (vi- nylpyrolidone) (PVP) NFs loaded with Au NPs. For application of nanodevice fabrication PAH NFs were made electrically conductive by blending electrospinning $\mathrm{PAH}$ NFs and loading Au NPs by UV irradiation technique. NFs loaded with Au NPs of diameter 100 - 150 nm were obtained [18]. A number of articles have been published on the preparation of metallic NPs blended with polysaccharides cellulose [19,20]. Chitin macro-molecule has been selected in the present work to blend with Au NPs since the compound is a natural biocompatible, biodegradable, nontoxic, and bioadsorbable polysaccharide mainly extracted from crab and prawn shells and second most abundant biomolecule after cellulose. Blending of nanometals with chitin has added further dimensions to already existing chitin application in wound healing [21]. On the other hand, the applications of NPs have been widened when they are embedded on CNFs in the field of medicine [22], medical imaging [23], and electrooptical devices [24].

\section{Experimental}

\subsection{Materials}

Precursor salts hydrogen tetrachloroaurate (III) trihydrate $\left(\mathrm{HAuCl}_{4} \cdot 3 \mathrm{H}_{2} \mathrm{O}\right)$ and $\mathrm{NaBH} 4$ were from Wako Pure Chemical Industries Ltd. Triblock copolymer poly (methyl vinyl ether) PMVE stabilizing agent was from Aldrich. Ethanol was obtained from KANTO CHEMI CAL CO., INC. Chitin powder from crab shells was purchased from NACALAI TESQUE, INC. Acetic acid was procured from Kanto chemical. Tricyclodecane dimethylol dimethacrylate (TCDDMA) was obtained from Chisso Corporation. Photoinitiator 2-hydroxy-2-methyl-propiophenone was from Tokyo Kasei Kogyo Co. Ltd.

\subsection{Preparation of CNFs}

Commercial pure chitin powder was dissolved in water at $1 \mathrm{wt} \%$ content and acetic acid was added to adjust $\mathrm{pH}$ to 3. The mixture was stirred over night at room temperature. Wet chitin cake was then obtained by vacuum filterring the slurry. Chitin cake was again dissolved in water. In second step the chitin concentration was higher at $1.3 \mathrm{wt} \%$ then $\mathrm{pH}$ was again adjusted to 3 by adding acetic acid for the preparation of mixuture for fibrillation. Slurry was passed through a grinder (MKCA6-3; Masuko Sangyo Co., Ltd.) at $1500 \mathrm{rpm}$. After passing slurry through grinder by two passes a high viscous gel of CNFs was obtained and used in this study. The yield of chitin in CNFs was obtained by dry weight of CNFs and it was $1.18 \mathrm{wt} \%$.

\subsection{Preparation of Au NPs in Soluble Polymer}

Precursor salt stock solution (0.05 M) was prepared in 
water. The measured amount of Au salt stock solution was added to triblock copolymer PMVE (0.6 wt\%) water dispersion to give a final Au salt concentration $10^{-3} \mathrm{M}$. $\mathrm{Au}$ salt was reduced in polymer dispersion by addition of freshly prepared NaBH4 stock solution $(0.1 \mathrm{M})$ in ethanol. All preparations were conducted in ambient conditions at $25^{\circ} \mathrm{C}$ and atmospheric pressure.

\subsection{Preparation of CNF-Au NPs Composites}

First diluted (16 wt\%) dispersion of CNFs were prepared in water by stirring and sonication then preorganized $\mathrm{Au}$ NPs in soluble polymer were poured in diluted NF suspension in equal proportion. The mixture were stirred and sonicated for 30 minutes each at room temperature. Sheets or thin films of neat CNFs and composite were prepared by taking the amount of composite that contains 20 g CNFs in a beaker. Preparations were vacuum stirred for $30 \mathrm{~min}$ to remove air bubble then filtered under vacuum on Teflon filter and washed three times with ethanol. Thus a thin composite film was prepared, film was then pressed dried at $100^{\circ} \mathrm{C}$ to remove moisture, finally a dried film of composite was obtained. Flakes and powder of composite were prepared by removing water from CNF-Au NPs suspension in a vacuum evaporator. pieces and ground to prepare flakes and powder, respectively.

\subsection{Recording Digital Images of Preparations}

When Au precursor salt was reduced in soluble polymer, color change and physical state of composite such as clear transparent or suspension was monitored visually by naked eyes and images of preparations were recorded digitally using a camera of model Sony (Cybershot DSCT1). Change occurred in color of Au salt from light golden to wine red color when salt reduced to nanometallic Au NPs in polymerwater medium. The pictures of contrasting color of neat CNFs and CNFs-Au NPs blended composite suspension, settled precipitate, and thin film were also recorded. Reporting of these pictures in the paper are important for readership to understanding better the stepwise phenomena of preparations.

\subsection{Recording of Absorption Spectra}

A Jasco V 570 spectrophotometer was used to record UV-vis spectra of neat polymer-water, Au NPs in polymer solution, and targeted CNFs-Au NPs composite suspension.

\subsection{Field Emission-Scanning Electron Micrograph (FE-SEM) Measurement}

FE-SEM images of neat CNF and Au NPs loaded CNF sheet were recorded by apparatus JSM-6701 F, JEOL, Ltd. after coating with $2 \mathrm{~nm}$ platinum layer by an ion sputtering coater.

\subsection{X-Ray Diffraction Measurement}

A Rigaku, UltimaIV X-ray diffrectometer operating at 40 $\mathrm{kV}$ and $30 \mathrm{~mA}$ was used to record X-ray differection pattern of the composite sheet in wide angle mode in the range $30^{\circ}-90^{\circ} /$ two theta value vs count per second.

\subsection{Trasnmission Electron Micrograph (TEM)}

Hitachi H-800 microscope operating at $200 \mathrm{kV}$ was used for TEM recording of Au NPs in polymer-water solution and CNFs-polymer blend were done by diluting the NPs by water and loading a drop of the diluted solution on carbon-copper grid. Grid was left overnight for drying then mounted to TEM holder for micrograph recording. TEM determined the size, shape, and dispersity of the particles.

\section{Results and Discussion}

\subsection{Preparation of Pre-Organized Au NPs}

Paste obtained after removal of water was lyophiliIznedoutropr. In our previous article [25], we have prepared Au NPs get a dried lump of composite. Lump was broken into from Au precursor salt in polymer PMVE (0.6 wt\%) water medium. Golden yellow color Au salt changed to wine red color preparation when reduced by $\mathrm{NaBH} 4$. Wine red color is of Au NPs as confirmed by XRD, TEM, ICP, EDX, and UV-vis measurements [26,27].

\subsection{Fibrillation of Chitin}

CNFs were prepared from commercial crab shell dry chitin powder as per the method described by Ifuku et al. [1]. In brief, $1 \mathrm{wt} \%$ chitin powder was dissolved in $\mathrm{pH} 3$ acidic water by adding acetic acid. The mixture was stirred at room temperature over night. Slury was vacuum filtered to obtain wet chitin cake. Chitin cake was dissolved in water again, acetic acid was added to level a $\mathrm{pH}$ 3. This chitin-water slurry was ready for fibrillation in mechanical grinder. Two passes of grinding were performed, finally CNFs in the form of gel were obtained and used in this research. For confirmation of fibrillation FE-SEM of CNFs sheet was recorded. The fiber width was 25 - 40 nm (Figure 1).

\subsection{Preparation of CNFs-Au NPs Blend Composite}

A diluted (16 wt\%) CNFs suspension (Figure 2(a)) of chitin content 0.18 wt\% was prepared. Au NPs (Figure 2(b)) of $\mathrm{Au}(0)$ content $5 \times 10^{-4} \mathrm{M}$ stabilized in polymer were then added to NF suspension in equal proportion to 


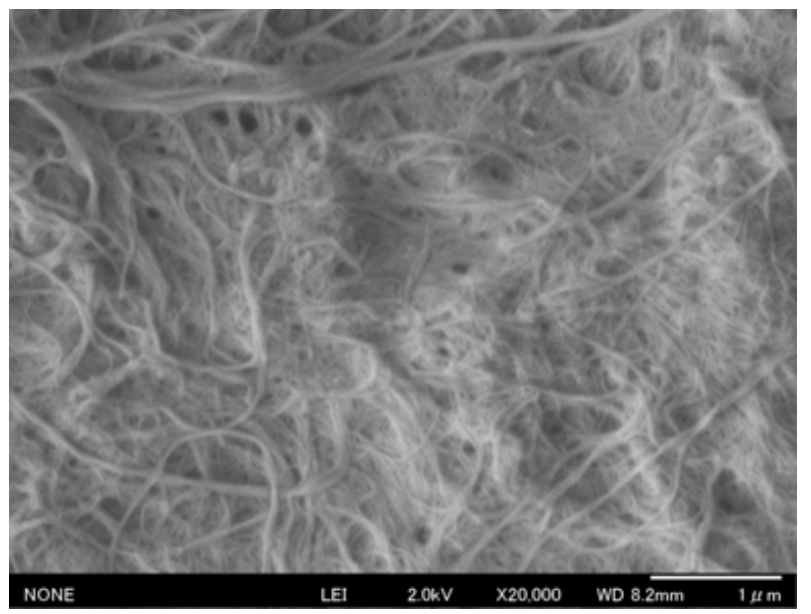

Figure 1. FE-SEM image of two cycles pass grinding of; scale: $1000 \mathrm{~nm}$.

get a light chocolate color (Figure 2(c)) of CNFs-Au NPs suspension. On keeping unstirred the NFs-NPs suspension for $4 \mathrm{~h}$, phase separation (Figure 2(d)) of composite occurred. Supernatant mother liquor is colorless and does not has any Au NPs, indicative that all Au NPs mass transferred to more electronegative moieties of chitin molecules shedding the capping shell of polymer. The net chitin and gold metal content ratio in molecular massto-atomic mass of the composites was 60:1 respectively. The composite flakes (Figure 2(e)) and powder (Figure 2(f)) were also prepared as described in experimental section. They have characteristics color of maroon and magenta, respectively.

\subsection{Preparation of Neat CNFs, CNFs-Au NPs, and Transparent CNFs and CNFs-Au NPs Films}

Figures 3(a) and (b) are the thin films of neat CNFs and blended CNFs-Au NPs composites. Neat CNFs sheet was buff brown while blend of CNFs-Au NPs sheet was apparently reddish black (maroon) color in appearance. These $70 \mathrm{~m}$ thin films were prepared as described in experimental section. Neat CNFs (Figure 3(a)) and CNFAu NPs composite films (Figure 3(b)) had \%transmittance (spectra not shown). When above two films were soaked in TCDDMA resin and polymerized by photoinitiator 2-hydroxy-2-methylpropiophenone under UV irradiation for 10 minutes after sandwiching the film between two glass plates. The soaked and cured films became transparent (Figures 3(c) and 3(d)). TCDDMA treated and cured neat CNFs and CNF-Au NPs composite films had a transmittance of $80 \%$ and $68 \%$ at $800 \mathrm{~nm}$ visible light (spectra are not shown). The phenomena of all four thin films was shown clearly for readership by enveloping a flower by films that can show the transparency apparently that developed by treating the films and subsequent curing.

\subsection{Neat CNFs and CNFs-Au NPs Composite Charac-Terization}

\subsubsection{Visible Spectroscopic Measurements}

Stepwis Stepwise visible spectroscopic recording is shown in Figure 4 of final CNFs-Au NPs composites preparation in solution and suspension forms. Spectra (a) is of the aqueous supernatant mother liquor separated from composite suspension when the preparation (preparations referred to Figure 2 captions) was left for $4 \mathrm{~h}$ (preparation 1d). In precipitate at the bottom of tube is CNFs-Au NPs settled wet composite. Since the spectra is a flat base line with no trace of Au NPs is the evidence that all NPs shifted to polar moieties of CNFs. Spectra of CNFs-polymer (b) has no band in the region has low intensity than the neat CNFs suspension spectrum (c) and (preparation 1(a) which also has no peak but absorbance is higher than spectrum (b) due to polymer blend with CNFs decreased the turbidity of the suspension. Spectrum (d) is of CNFs-Au NPs composite (preparation 1c), presence of Au NPs in the composite is confirmed by the presence of plasmon resonance band at $\lambda=520 \mathrm{~nm}$ charac teristic of Au NPs. The last spectrum (e) is of $\mathrm{Au}$ NPs in polymer-water solution (preparation1b). In spectrum (e) a sharp intense band at $\lambda=520 \mathrm{~nm}$ was noticed which is the characteristic of small Au NPs (4.4 nm $(\sigma=$ 1.2)) in the solution.

\subsubsection{TEM Investigation of CNFs-Au NPs Suspension Composite}

Figure 5(a) is TEM image of composite in liquid suspension form (preparation 1c). The composite (Figure 1(c)) has a chocolate cake color appearance. The composite suspension was diluted with water for loading a drop on carbon-copper grid for TEM observation. In histogram (Figure 5(b)) mainly four different types of NPs were noticed of sizes: $4.5,7.5,12$, and $22.5 \mathrm{~nm}$ diameter with $>50 \%$ particles of $12 \mathrm{~nm}$ diameter and average particles size $11.2 \mathrm{~nm}(\sigma=7.8)$.

\subsubsection{FE-SEM Measurement}

Figure 6 is the FE-SEM image of Au NPs loaded CNFs, in the picture white tiny dots siting on NFs' surface are $\mathrm{Au}$ NPs which are visible all over the CNFs film. The average size of Au NPs supported on the CNFs is $19 \mathrm{~nm}(\sigma=7)$. The size of NPs in film is large due to limiting resolution of SEM compared to observed in liquid CNFs suspension observed by TEM. The other reason of larger particles is that particles are stacked one over the other making larger crystals. While in diluted CNFs suspendsion particles are well dispersed thus size of particles is small in TEM image. 


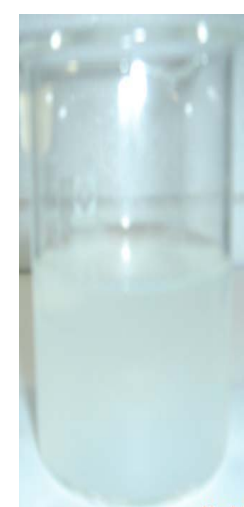

(a)

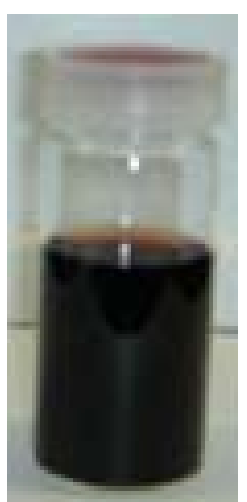

(b)

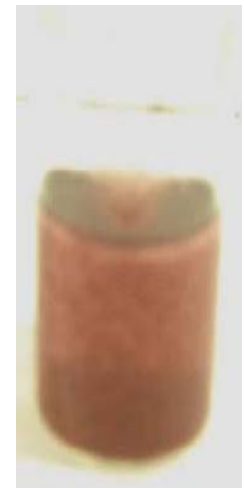

(c)

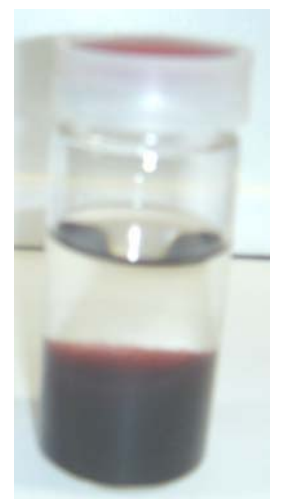

(d)

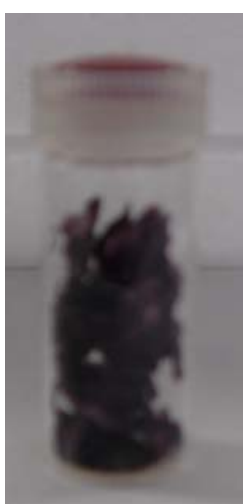

(e)

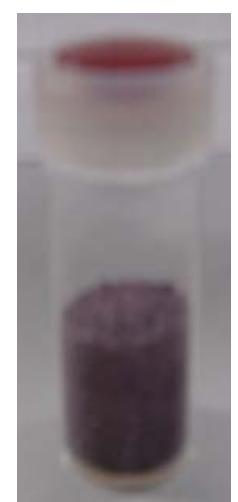

(f)

Figure 2. Stepwise preparation of CNFs-Au NPs composites; (a) diluted CNFs suspension; (b) pre-organized Au NPs in polymer; (c) blend of CNFs-Au NPs stirred preparation; (d) phase separated blend in 4 h, no stirring; (e) flakes of CNFs-Au NPs; (f) CNFs-Au NPs powder.

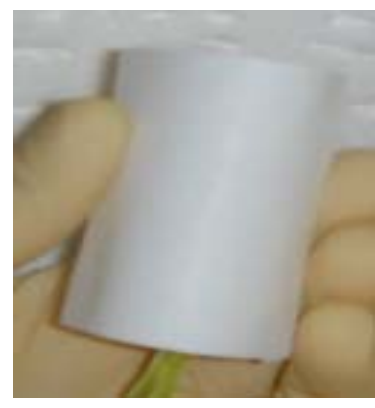

(a)

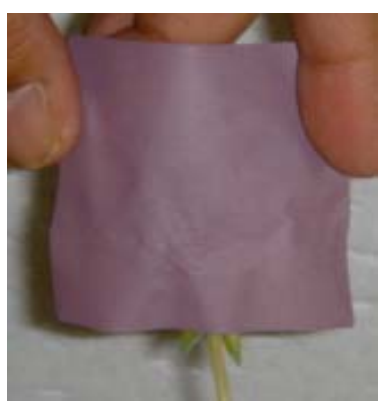

(b)

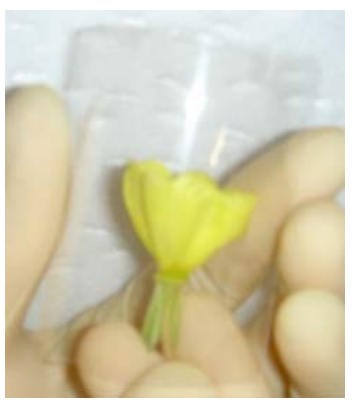

(c)

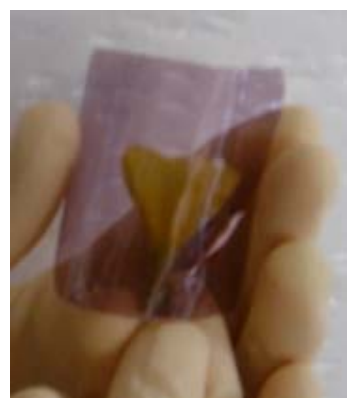

(d)

Figure 3. Optical Property of (a) neat CNFs; (b) CNFs-Au NPs composite film before treatment; (c) neat CNFs and (d) CNFs-Au NPs composite film after treatment. Treatment was conducted by impregnating film with tricyclodecane dimethylol dimethacrylate (TCDDMA) and subsequent polym-erization by photoinitiator 2-hydroxyl-2-methylpropiophenone by UV irrediation.

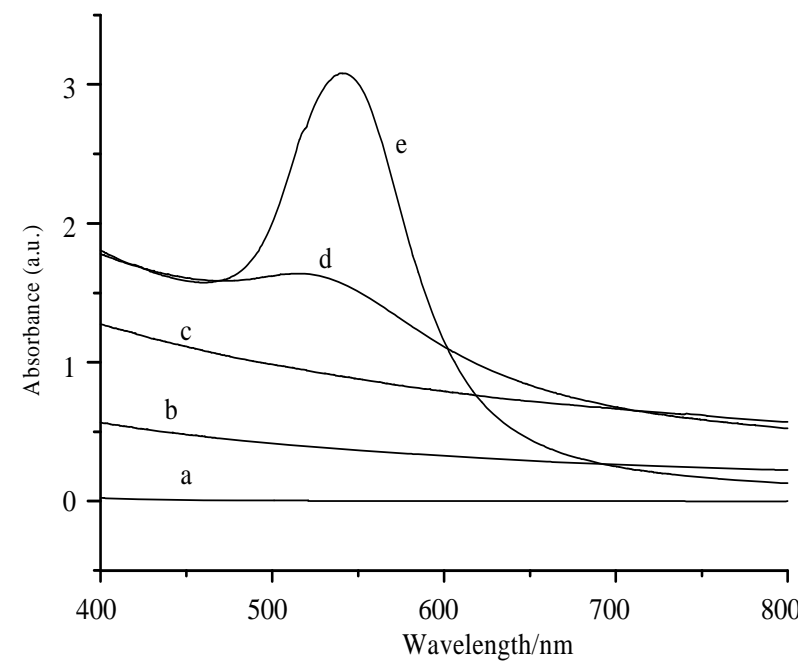

Figure 4. Visible spectra of stepwise preparation of CNFsAu NPs composite of the preparation as shown in Figure 2; a. supernatant mother liquor (preparation; Figure 2d), b. CNFs-polymer suspension base line, c. Neat CNFs suspendsion (preparation; Figure 2(a)) CNF-Au NPs composite (preparation; Figure 2(c)), e. Au NPs in polymer (preparation; Figure 2(b)).

\subsubsection{XRD of Composite}

XRD pattern of CNFs-Au NPs film is shown in Figure 7. Spectrum has been divided in chitin XRD bands (Figure 7(a)) which matched with well known chitin pattern in two theta range $5^{\circ}-30^{\circ}$. The other half had Au metallic pattern finger prints (Figure 7(b)) in the range $30^{\circ}-90^{\circ}$. Thus XRD. recording confirmed the composition of chitin-Au metallic composition. The band obtained at positions: $9.6^{\circ}, 19.7^{\circ}$, and $23.7^{\circ}$ on two theta scale are the characteristic of chitin of planes: 020,110 , and 130 , respectively. The band of highest intensity at $19.7^{\circ}$ position is characteris-ticof high crystalline structure of chitin. While in second half, the Au metallic bands are at positions: $38.8^{\circ}, 44.3^{\circ}, 65.1^{\circ}$, and $77.1^{\circ}$ belongs to planes: 111 , 200,220 , and 311 , respectively. At $38.8^{\circ}$ two theta value band intensity is highest showed the Au metal maintained high crystallinity even in the form of organic-inorganic hybrid composite.

\section{Evidence of Polymer and Au NPs Mass Transfer onto CNFs Surface}

This research is based on phase (polymer and Au NPs) 


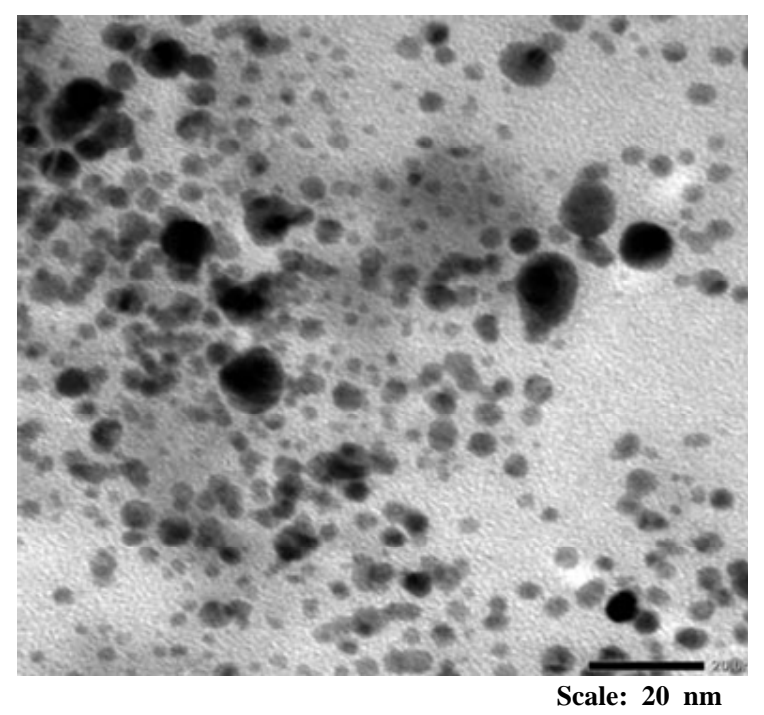

(a)

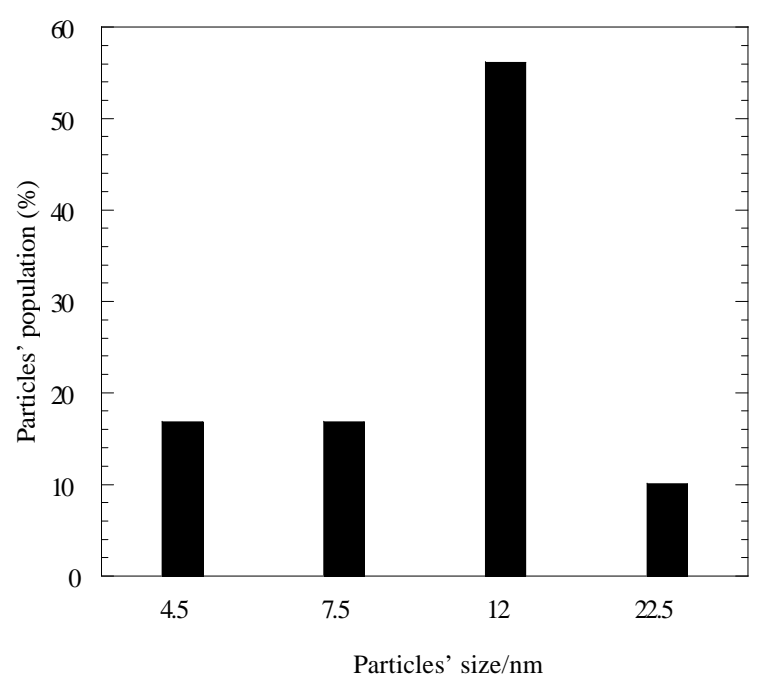

(b)

Figure 5. (a) TEM image of Au NPs in CNFs-Au NPs composite, (b) is the histogram of the TEM microgram of TEM image. Average particles' size is $11.2 \mathrm{~nm}(\sigma=7.8)$.

transfer from water-polymer bulk solution to more polar moieties of CNF surface. All types of composites of CNFs-Au NPs as mentioned in text were prepared on this mass transfer phenomenon. When wine red color preorganized Au NPs in polymer was mixed to CNFs dispersion, 56\% polymer and all Au NPs transferred to more electronegative CNFs surface, leaving supernatant mother liquid colorless. While CNFs suspension, polymer and $\mathrm{Au}$ NPs settled as chocolate cake at the bottom of the tube (Figure 2(d)) As verified by comparing band intensities of UV-vis spectra (Figure 8) of polymer before blending with NFs and after mixing with NFs, it was confirmed that $56 \%$ of polymer and $100 \%$ NPs transferred from water to CNFs suspension.

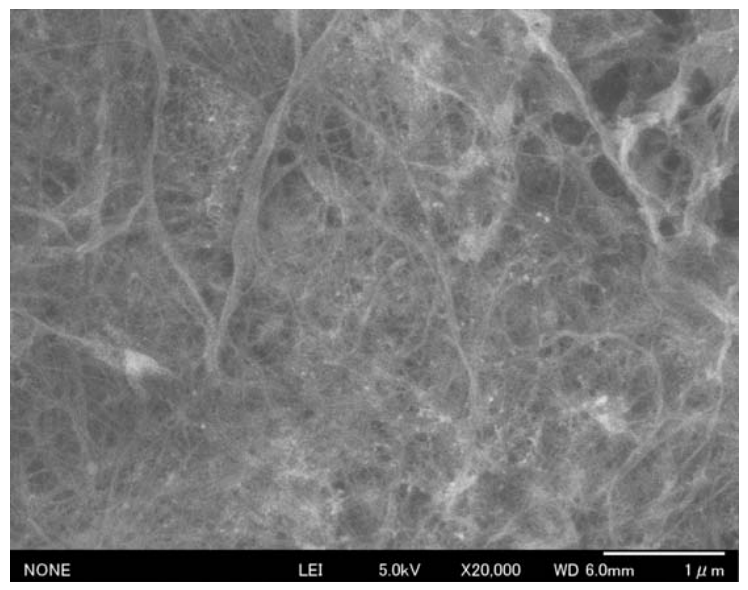

Figure 6. FE-SEM Micrograph of CNFs-Au NPs film; the fibres' width is 20 - $40 \mathrm{~nm}$, Au NPs particles of $19 \mathrm{~nm}(\sigma=7)$ diameter are clearly visible on the surface of fibers.

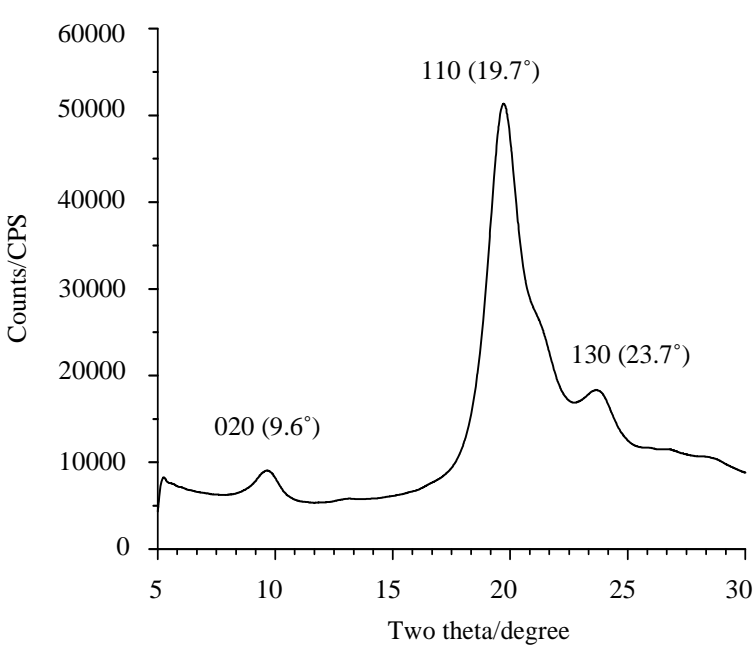

(a)

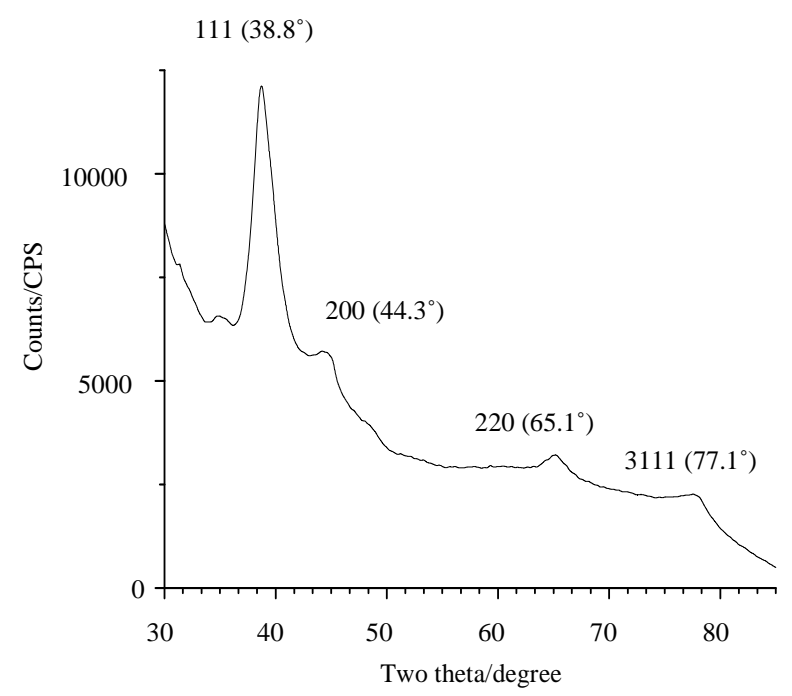

(b)

Figure 7. XRD pattern of chitin (a) and Au metallic (b) parts of composite film. 


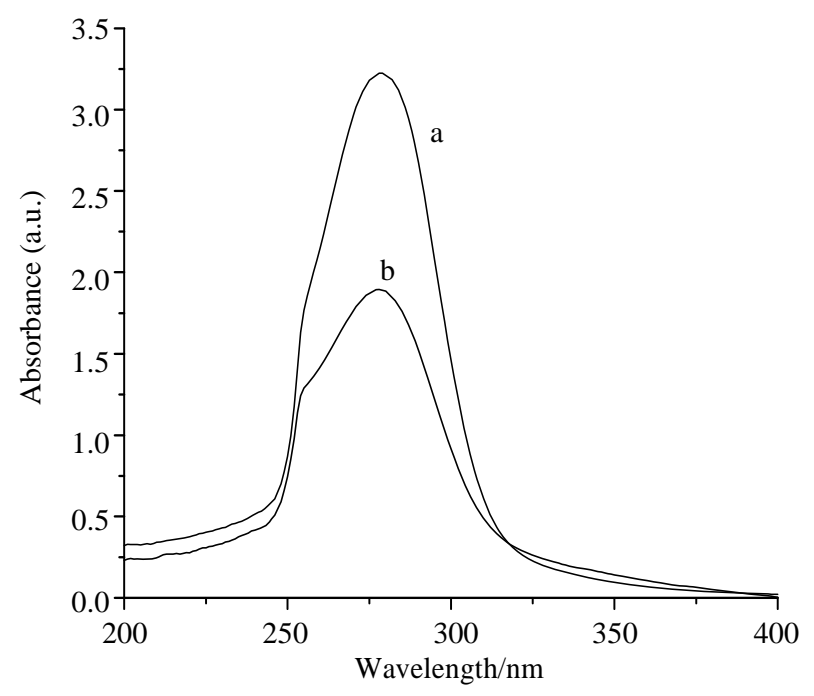

Figure 8. UV-vis spectra (a) of polymer before adding CNFs and (b) after adding CNFs.

\section{Mechanism of Loading Au NPs onto CNFs}

When preorganized Au NPs in polymer solution were mixed with CNFs dispersion in equal proportion, all $\mathrm{Au}$ NPs and and 64\% polymer (Figure 8) were mass transferred from water-polymer solution to more polar chitin mole-cules moieties. Since the mother liquor on filtration of CNF-polymer-Au NPs was colorless transparent liquid thus all Au NPs entangled onto more polar chitin moieties compared to polymer molecules. Au NPs shed the shell of polymer and get attached to chitin in CNFs and remain in CNFs when filtered on Teflon filter under vacuum, thus Au NPs and CNFs make thin of CNF-Au NPs composite film. Due to blending of Au NPs in CNFs the composite film appears reddish (Figures 3(b) and (d)).

\section{Conclusions}

Second most abundant biomaterial on earth chitin has been atomized into CNFs of $25-40 \mathrm{~nm}$ width and high aspect ratio. The applications of CNFs were further explored by preparing organic-inorganic type nanocomposite by preparing organic-inorganic type nanocomposite in a number of physical forms suspension, thin film (70 $\mathrm{m})$, flakes and powder. Apart from non-toxic and biodegradable, composite preparation method has a number of advantages over other published methods till now. Composite can be prepared in short time rather taking days under ambient conditions. Preparation avoids use of any toxic organic solvents. The materials molded in suspension, flakes, powder, and sheet or thin film can serve a variety of purposes as suitable to applications from cosmetic, pharmaceuticals, catalysts, electrical, electronic and optical devices. A number of methods visual ob- servation, UV-vis, TEM, FE-SEM, XRD, and were employed to prepare and characterize the composite. XRD pattern confirmed the chitin and Au metallic contents in the composite. Visual observation and UV-vis spectra confirmed that $56 \%$ polymer and $100 \%$ NPs phase transferred from polymer-water solution to CNFs sus- pension. Phase transfer phenomena caused CNFs-Au NPs composite formation. Neat CNFs and CNFs-Au NPs films became $80 \%$ and $68 \%$ transparent, respectively when impregnated with TCDDMA resin and poly- merized by photoinitiator 2-hydroxy-2-methylpropioph- enone under UV irradiation.

\section{REFERENCES}

[1] S. Ifuku, M. Nogi, K. Abe, M. Yoshioka, M. Morimoto, H. Saimoto and H. Yano, "Preparation of Chitin Nanofibers with a Uniform Width as Chitin from Crab Shells," Biomacro-Molecules, Vol. 10, No. 6, 2009, pp. 15841588.

[2] S. Ifuku, M. Nogi, M. Yoshioka, M. Morimoto, H. Yano and H. Saimoto, "Simple Preparation Method of Chitin Nanofibers with a Uniform Width of 10-20 nm from Prawn Shell under Neutral Conditions," Carbohydrate Polymers, Vol. 84, No. 2, 2011, pp. 762-764.

[3] N. K. Gopalan and A. Dufresne, "Crab Shell Chitin Whisker Reinforced Natural Rubber Nanocomposites. 1. Processing and Swelling Behavior,” Biomacromolecules, Vol. 4, No. 3, 2003, pp. 657-665.

[4] K. Azuma, T. Osakia, T. Wakuda, S. Ifuku, H. Saimoto, T. Tsuka, T. Imagawa, Y. Okamoto and S. Minami, "Beneficial and Preventive Effect of Chitin Nanofibrils in a Dextran Sulfate So-Dium-Induced Acute Ulcerative Colitis Model," Carbohydrate Polymers, Vol. 87, No. 2, 2012, pp.1399-1403.

[5] Y. Sueyoshi, T. Hashimoto, M. Yoshikawa and S. Ifuku, "Chitin Nanofiber Membranes for Chiral Separation," Sustainable Agriculture Research, Vol. 1, No. 1, 2012, pp. 42-47. doi:10.5539/sar.v1n1p42

[6] S. Ifuku, A. Ikuta, T. Hosomi, S. Kanaya, Z. Shervani, M. Morimoto and H. Saimoto, "Preparation of Polysilsesquioxane-Urethaneacrylate Copolymer Film Reinforced with Chitin NanofiBers," Carbohydrate Polymers, Vol. 89, No. 3, 2012, pp. 865-869.

[7] S. Ifuku, M. Tsuji, M. Morimoto, H. Saimoto and H. Yano, "Synthesis of Silver Nanoparticles Templated by TEMPO-Mediated Oxidized Bacterial Cellulose Nanofibers," Biomacro-Molecules, Vol. 10, No. 9, 2009, pp. 2714-2717. doi:10.1021/bm9006979

[8] S. Ifuku, K. Yamada, M. Morimoto and H. Saimoto, "Nanofibrillation of Dry Chitin Powder by Star Burst System,” Journal of Nanomaterials, Vol. 2012, 2012. doi:10.1155/2012/645624

[9] S. Ifuku and H. Saimoto, "Chitin Nanofibers: Preparations, Modification, and Applications,” Nanoscale, Vol. 4, No. 11, 2012, pp. 3308-3318.

[10] S. Ifuku, M. Iwasaki, M. Morimoto and H. Saimoto, "Po- 
Lymerization of Acrylic Acid onto Chitin Nanofibers to Improve Dispersibility in Basic Water," Carbohydrate Polymers, Vol. 90, No. 1, 2012, pp. 623-627. doi:10.1016/j.carbpol.2012.05.087

[11] K. Azuma, T. Osaki, T. Wakuda, S. Ifuku, M. Saimoto, T. Tsuka, T. Imagawa, Y. Okamoto and S. Minami, "Beneficial of and Preventive Effect of Chitin Nanofibers in a Dextran Sulfate Sodium Induced Acute Ulcerative Colitis Model," Carbohydrate Polymers, Vol. 87, No. 2, 2012, pp. 1399-1403.

[12] S. Ifuku, Z. Shervani, M. Mrimoto and M. Saimoto, "Processing, Properties, and Applications in Biopolymer Nanocom-Posites,” In: A. Dufresne, S. Thomas and L. A. Pothan, Eds., Wiley Series on Polymer Engineering and Technology, John Wiley \& Sons, Inc., Hoboken, 2012.

[13] S. Ifuku, Z. Shervani and H. Saimoto, "Preparation, Modification and Application of Chitin Nanofibers," In: W. D. Li and X. P. Wang, Eds., Nanofibers: Synthesis, Properties, and Applications, Nova Scientific Publishers, New York, 2012.

[14] S. Rana, A. Bajaj, R. Mout and V. M. Rotello, "Monolayer Coated Gold Nanoparticles for Delivery Applications," Advanced Drug Delivery Reviews, Vol. 64, No. 2, 2011, pp. 200-216.

[15] D. T. Nguyen, D. Kim and K. Kim, “Controlled Synthesis and Biomolecular Probe Application of Gold Nanoparticles,” Micron, Vol. 42, No. 3, 2011, pp. 207-227.

[16] A. E. Deniz, H. A. Vural, B. Ortac and T. Uyar, "Gold Nanoparticles/Polymer Nanofibrous Composites by Laser Ablation and Electrospinning," Materials Letters, Vol. 65, No. 19, 2011, pp. 2941-2943.

[17] S. Kundu, R. S. Gill and R. F. Saraf, "Electrospinning of PAH Nanofibers and Deposition of Au NPs for Nanodevice Fabrication" Journal of Physical Chemistry C, Vol. 115, No. 32, 2011, pp. 15845-15852.

[18] A. B. Etame, C. A. Smith, W. C. W. Chan and J. T. Rutka, "Design and Potential Application of Pegylated Gold Nanoparticles with Size-Dependent Permeation through Brain Microvas-Culature," Nanomedicine: Nanotechnology, Biology, and Medicine, Vol. 7, No. 6, 2011, pp. 992-1000. doi:10.1016/j.nano.2011.04.004

[19] S. Ifuku, M. Tsuji, M. Morimoto, H. Saimoto and H. Yano, "Synthesis of Silver Nanoparticles Template by Tempo-Mediated Oxidized Bacterial Cellulose Nano- fi- bers,” Biomacromoles, Vol. 10, No. 8, 2009, pp. 27142717.

[20] P. Dallas, V. K. Sharma and R. Zboril, "Silver Polymeric Nanocomposites as Advanced Antimicrobial Agents: Classification, Synthetic Paths, Applications, and Perspectives," Advances in Colloid and Interface Science, Vol. 166, No. 1-2, 2011, pp. 119-135.

[21] K. Azuma, T. Osaki, T. Wakuda, S. Ifuku, H. Saimoto, T. Tsuka, T. Imagawa, Y. Okamoto and S. Minami, "Beneficial and Preventive Effect of Chitin Nanofibrils in a Dextran Sulfate Sodium-Induced Acute Ulcerative Colitis Model," Carbohydrate Polymers, Vol. 87, No. 2, 2012, pp. 1399-1403. doi:10.1016/j.carbpol.2011.09.036

[22] K. H. L. Kwan, X. Liu, M. K. T. To, K. W. K. Yeung, C. Ho and K. K. Y. Wong, "Modulation of Collagen Alignment by Nanoparticles Results in Better Mechanical Properties in Wound Healing," Nanomedicine: Nanotechnology, Biology, and Medicine, Vol. 7, No. 4, 2011, pp. 497-504.

[23] D. P. Cormode, P. A. Jarzyna, W. J. M. Mulder and Z. A. Fayad, "Modified Natural Nanoparticles as Contrast Agents for Medical Imaging," Advanced Drug Delivery Reviews, Vol. 62, No. 3, 2010, pp. 329-338.

[24] W. Yoon, K. Jung, J. Liu, T. Duraisami, R. Revur, F. T. Teixeira, S. Sengupta and P. R. Berger, "Plasmonenhanced Optical Absorption and Photocurrent in Organic Bulk Hetero-Junction Photovoltic Devices Using Self-Assembled Layer of Silver Nanoparticles," Solar Energy Materials and Solar Cells, Vol. 94, No. 2, 2010, pp. 128- 132.

[25] Z. Shervani and Y. Yamamoto, "Soluble Polymer Directed Controlled Synthesis of Gold Nanoparticles: Effect of Cloud Point, Reducing Agents and Mixing Methods on the Nanoparticles Preparation," International Journal of Nanoscience, Vol. 11, No. 1, 2012, pp. 57-66.

[26] Z. Shervani and Y. Yamamoto, "Size and Morphology Controlled Synthesis of Gold Nanoparticles in Green Solvent,” Materials Letters, Vol. 65, No. 1, 2011, pp. 9295.

[27] Z. Shervani and Y. Yamamoto, "Carbohydrate Directed Synthesis of Silver And Gold Nanoparticles: Effect of the Structure of Carbohydrates and Reducing Agents on the Size and Morphology of the Composites," Carbohydrate Research, Vol. 346, No. 5, 2011, pp. 651-658. 